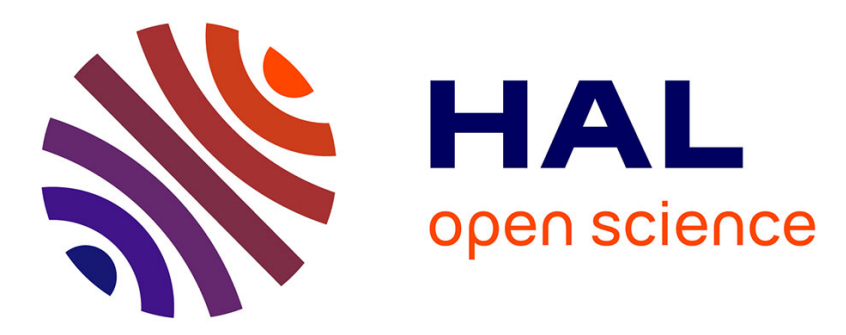

\title{
Homology of the Lie algebra of vector fields on the line with coefficients in symmetric powers of its adjoint representation
}

Vladimir Dotsenko

\section{- To cite this version:}

Vladimir Dotsenko. Homology of the Lie algebra of vector fields on the line with coefficients in symmetric powers of its adjoint representation. Functional Analysis and Its Applications, 2006, 40 (2), pp.91-96. 10.1007/s10688-006-0015-2 . hal-02482167

\section{HAL Id: hal-02482167 https://hal.science/hal-02482167}

Submitted on 17 Feb 2020

HAL is a multi-disciplinary open access archive for the deposit and dissemination of scientific research documents, whether they are published or not. The documents may come from teaching and research institutions in France or abroad, or from public or private research centers.
L'archive ouverte pluridisciplinaire HAL, est destinée au dépôt et à la diffusion de documents scientifiques de niveau recherche, publiés ou non, émanant des établissements d'enseignement et de recherche français ou étrangers, des laboratoires publics ou privés. 


\title{
HOMOLOGY OF THE LIE ALGEBRA OF VECTOR FIELDS ON A LINE WITH COEFFICIENTS IN SYMMETRIC POWERS OF ITS ADJOINT REPRESENTATION
}

\author{
V. V. DOTSENKO
}

\begin{abstract}
Aвstract. We compute the homology of the Lie algebra $W_{1}$ of (polynomial) vector fields on a line with coefficients in symmetric powers of its adjoint representation. We also list the results obtained so far for the homology with coefficients in tensor powers and, in turn, use them for partially computing the homology of the Lie algebra of currents on a line with values in $W_{1}$.
\end{abstract}

KEYwords. Lie algebras of vector fields, Lie algebra (co)homology.

\section{Introduction.}

Denote by $W_{1}$ the Lie algebra of polynomial vector fields on a line, i.e. the algebra of vector fields $f(x) \frac{d}{d x}$, where $f(x)$ is a polynomial. The vector fields $e_{i}=x^{i+1} \frac{d}{d x}(i \geq-1)$ form a basis in this algebra, and the commutation relations are $\left[e_{i}, e_{j}\right]=(j-i) e_{i+j}$. We compute the homology of the Lie algebra $W_{1}$ with coefficients in modules $S^{k} W_{1}$, i.e. the symmetric powers of its adjoint representation. Namely, we prove the following results.

Main theorem. Let $k>0$. Then

(i) $H_{2}\left(W_{1}, \mathfrak{g l}_{1}, S^{k} W_{1}\right)=\mathbb{C}, H_{i}\left(W_{1}, \mathfrak{g l}_{1}, S^{k} W_{1}\right)=0$ for $i \neq 2$,

(ii) $H_{2}\left(W_{1}, S^{k} W_{1}\right)=H_{3}\left(W_{1}, S^{k} W_{1}\right)=\mathbb{C}, H_{i}\left(W_{1}, S^{k} W_{1}\right)=0$ for $i \neq 2,3$.

This proves the $n=1$ case of the conjecture on the homology of the algebra $W_{n}$ with coefficients in symmetric powers of its adjoint representation stated by B. Feigin and B. Tsygan [1].

This computation is based on a partial computation of the homology (with the same coefficients) for a subalgebra of $W_{1}$ consisting of vector fields which vanish twice at $x=0$. (This algebra is denoted by $L_{1}$; the elements $e_{i}$ with $i \geq 1$ form a basis for this subalgebra. More generally, the subalgebra spanned by the elements $e_{i}$ with $i \geq k$ is denoted by $L_{k}$.) In general, the homology of the Lie algebra $L_{1}$ with nontrivial coefficients are more complicated but in our case it turns out that $L_{1}$-modules $S^{k} W_{1}$ are free in low degrees. (To be more precise, this means that there exists a (homogeneous) homomorphism from a free $L_{1}$-module onto $S^{k} W_{1}$, which has a trivial kernel on all homogeneous components whose degree is sufficiently small; see Corollary 2 below.) The homology with coefficients in a free module is known; if a module is free in low degrees, one can easily compute the corresponding graded component of its homology. Methods of studying the homology of $W_{1}$ on the basis of information about the homology of $L_{1}$ with the same coefficients are quite traditional (e.g., see [2]).

The text is organised as follows. In the first part we study the symmetric powers in low degrees as $L_{1}$ modules. This can be done by means of some linear algebra. (Actually, the point is that matrices of certain operators are upper-triangular.) In the second part of the paper, we use these results to obtain information about the homology of $W_{1}$. This could be done by standard homological algebra techniques. We finish the paper with a discussion of the case of tensor powers of the adjoint representation; in particular, we suggest a (conjecturally almost exact) lower bound for the homology.

I am grateful to my teacher Boris Feigin for stating the problem and for stimulating discussions. I am also grateful to the anonymous referee for numerous suggestions for improving the text.

The work of the author is partially supported by the grant of the President of the Russian Federation 2044.2003.2 and by the INTAS grant 03-3350. 


\section{LOW DEGREES}

We consider the standard grading on the Lie algebras $W_{1}$ and $L_{1}$, deg $e_{i}=i$. All the modules are supposed to be graded; the homomorphisms are assumed to be graded of degree zero.

Consider a natural ordering of the basis elements of $W_{1}: e_{i}>e_{j}$ for $i>j$. We take the standard monomial basis of $S^{k} W_{1}$ consisting of the elements $e_{i_{1}} e_{i_{2}} \cdot \ldots \cdot e_{i_{k}}\left(-1 \leq i_{1} \leq \ldots \leq i_{k}\right)$. Consider the following ordering of this basis: we drop all factors equal to $e_{-1}$ compare the monomials $e_{i_{1}} e_{i_{2}} \cdot \ldots \cdot e_{i_{l}}$ with $l \leq k$ and $0 \leq i_{1} \leq \ldots \leq i_{l}$ ) using the degree-lexicographic ordering: the monomial with larger $l$ is larger, and monomials having the same $l$ are compared lexicographically. According to the Poincare-Birkhoff-Witt theorem [3], the elements $E_{\alpha}:=e_{\alpha_{1}} e_{\alpha_{2}} \ldots e_{\alpha_{n}}$, where $\alpha$ runs over all multiindices $\left(\alpha_{1}, \ldots \alpha_{n}\right)$ such that $0<\alpha_{1} \leq \alpha_{2} \leq \ldots \leq \alpha_{n}$, form a basis in the universal enveloping algebra $U=U\left(L_{1}\right)$ for the Lie algebra $L_{1}$. For these elements we also consider the degree-lexicographic ordering. For an element $f \in S^{k} W_{1}$, we denote by $\widehat{f}$ its leading monomial with respect to the ordering introduced above.

Lemma 1. Let $E_{\alpha}<E_{1^{k+1}}=e_{1}^{k+1}$. Then

$$
\left.\widehat{E_{\alpha}\left(e_{-1}^{k}\right.}\right)=\frac{k !}{(k-n) !}(-1)^{n}\left(1+\alpha_{1}\right) \cdots\left(1+\alpha_{n}\right) e_{-1}^{k-n} e_{\alpha_{1}-1} \ldots e_{\alpha_{n}-1} .
$$

Proof. The proof is by a straightforward computation using the fact that the leading monomial is the monomial with the least number of occurrences of $e_{-1}$.

Corollary 1. Let $E_{\alpha}<E_{\beta}<e_{1}^{k+1}$. Then

$$
\widehat{E_{\alpha}\left(e_{-1}^{k}\right)}<\widehat{E_{\beta}\left(e_{-1}^{k}\right)}
$$

For each module $M$ we denote by $M[p]$ the module obtained from $M$ by the shift of grading by $p$, $M[p]_{i}:=M_{i-p}$.

Corollary 2. The $L_{1}$-module homomorphism $\rho: U[-k] \rightarrow S^{k} W_{1}$ mapping 1 to $e_{-1}^{k}$ is surjective. It is an isomorphism on the homogeneous components of degrees $-k, 1-k, \ldots, 0$ in $U[-k]$ and has one-dimensional kernel on the component of degree 1.

Proof. Surjectivity can be proved as follows: the leading monomials of the elements $\rho\left(E_{\alpha}\right)=E_{\alpha}\left(e_{-1}^{k}\right)$, where $\alpha$ runs over all multiindices $\left(\alpha_{1}, \ldots, \alpha_{l}\right)$ of length $l \leq k$ (so that $E_{\alpha}<e_{1}^{k+1}$ and we can use the previous corollary), are distinct. Moreover, all basis monomials of the symmetric power $S^{k} W_{1}$ occur among these leading monomials. (By Lemma 1, the leading monomial of the element $E_{\alpha}\left(e_{-1}^{k}\right)$ is, up to a constant factor, $e_{-1}^{k-l} e_{\alpha_{1}-1} \cdot \ldots \cdot e_{\alpha_{l}-1}$, and each basis monomial can be written in this form for a suitable $\alpha$.) It follows that any monomial from the basis belongs to the image of $\rho$.

The statement concerning the isomorphism can be established in a similar way. The dimensions of the graded components of degrees $-k, \ldots, 0$ in $U[-k]$ coincide with the corresponding dimensions in the symmetric power (since for each basis element $E_{\alpha}$ the length of of the multi-index $\alpha$ is at most $k$ ), and for the degree 1 the dimension differs by one (the only element for which the length of $\alpha$ is larger than $k$ is $e_{1}^{k+1}$ ).

For subsequent use, we choose and fix a homogeneous element $\phi \in U[-k]$ of degree 1 such that $\rho(\phi)=0$.

Corollary 3. The $L_{1}$-module $S^{k} W_{1}$ has a free resolution of the type

$$
0 \leftarrow S^{k} W_{1} \leftarrow U[-k] \leftarrow U[1] \oplus M^{1} \leftarrow M^{2} \leftarrow \ldots \leftarrow M^{i} \leftarrow \ldots
$$

Here all the modules $M^{i}$ are free, and have nonzero graded components only in degrees larger than one.

Proof. We follow the general scheme for constructing a minimal resolution for a module. A surjective homomorphism $\rho$ from $U[-k]$ to $S^{k} W_{1}$ is defined above. The next step is to construct a surjection from a free module onto its kernel. The kernel is generated by $\phi$ and finitely or infinitely many elements $\phi_{1}, \ldots, \phi_{s}, \ldots$ of larger degrees $n_{1}, \ldots, n_{s}, \ldots$ It follows that there exists a surjection from the free module $U[1] \oplus \bigoplus_{s>1} U\left[n_{s}\right]$ (the homomorphism maps the identity element of each summand to the corresponding generator), hence we may obtain the first module of the resolution by putting $M^{1}:=U[1] \oplus \bigoplus_{s \geq 1} U\left[n_{s}\right]$. The subsequent steps are similar; one should just note that the kernels of all the further homomorphisms may only contain elements of degrees larger than one. 
It is known [2] that homology of a Lie algebra $\mathfrak{A}$ with coefficients in a module $M$ can be computed in two different ways. First, this homology is equal to the homology of the Chevalley-Eilenberg complex $C_{*}(\mathfrak{H}, M)=\Lambda^{*}(\mathfrak{H}) \otimes M$. Second, in terms of the universal enveloping algebra one has $H_{*}(\mathfrak{A}, M)=\operatorname{Tor}_{*}^{U(\mathfrak{l})}(M, \mathbb{C})$. In the latter case, we can use our results on the free resolution. To write out the result, we recall that the homology of a graded Lie algebra $\mathfrak{A}$ with coefficients in a graded module $M$ inherits the grading. We denote by $H_{*}^{(p)}(\mathfrak{A}, M)$ the corresponding graded components.

\section{Proposition 1.}

$$
\begin{gathered}
H_{0}\left(L_{1}, S^{k} W_{1}\right)=H_{0}^{(-k)}\left(L_{1}, S^{k} W_{1}\right)=\mathbb{C}, \\
H_{1}^{(\leq 1)}\left(L_{1}, S^{k} W_{1}\right)=H_{1}^{(1)}\left(L_{1}, S^{k} W_{1}\right)=\mathbb{C}, \\
H_{>1}^{(\leq 1)}\left(L_{1}, S^{k} W_{1}\right)=0 .
\end{gathered}
$$

It is of interest to obtain some explicit formulae for the cycles representing these classes in the ChevalleyEilenberg complex. Let $\psi_{k}$ be the unique nontrivial class of the first homology in degree 1 .

Proposition 2. The element

$$
\sum_{i=0}^{k}(-1)^{i}\left(\begin{array}{c}
k+2 \\
i
\end{array}\right) e_{k+1-i} \otimes e_{-1}^{k-i} e_{0}^{i}
$$

of the Chevalley-Eilenberg complex $C_{*}\left(L_{1}, S^{k} W_{1}\right)$ is a representative of $\psi_{k}$.

Proof. A direct calculation shows that this element belongs to the kernel of the boundary map. It only remains to prove that this cycle is nontrivial. The easiest way to do this is to use the fact that the summand $e_{1} \otimes e_{0}^{k}$ never occurs in a boundary map of some element of the Chevalley-Eilenberg complex.

It is well known [2] that $L_{1}$ is generated by $e_{1}$ and $e_{2}$. Thus there exists also a representative of the type $e_{1} \otimes m_{1}+e_{2} \otimes m_{2}$. Let us give the corresponding explicit formulae, since they seem to be rather interesting. The main ingredient here is the "Casimir operator" $e_{-1} e_{1}-e_{0}^{2}$ for the subalgebra $\mathfrak{s l}_{2} \subset W_{1}$ spanned by $e_{-1}, e_{0}$, $e_{1}$.

Proposition 3. For $k=2 m$ the element

$$
e_{1} \otimes\left(e_{-1} e_{1}-e_{0}^{2}\right)^{m}
$$

is a representative for $\psi_{k}$, for $k=2 m+1$ the formula is sligtly more complicated:

$$
e_{2} \otimes e_{-1}\left(e_{-1} e_{1}-e_{0}^{2}\right)^{m}-(2 m+3) e_{1} \otimes e_{0}\left(e_{-1} e_{1}-e_{0}^{2}\right)^{m}+
$$

$$
+m e_{1} \otimes e_{-1}\left(e_{-1} e_{1}-e_{0}^{2}\right)^{m-1}\left(e_{-1} e_{2}-e_{0} e_{1}\right) .
$$

Proof. The reason for these cycles to be nontrivial is the same as above: a cycle containing $e_{1} \otimes e_{0}^{k}$ with a nonzero coefficient does not belong to the image of the boundary map.

\section{THE MAIN THEOREM}

To calculate the homology of $W_{1}$ with coefficients in $S^{k} W_{1}$ we use the Hochshild-Serre spectral sequence twice, in the absolute and in the relative case; we start with computation of the relative homology modulo the subalgebra $\mathfrak{g l}_{1}$ spanned by $e_{0}$ and then use the result of computation to prove the main theorem stated in the introduction.

Consider a chain of subalgebras $\mathfrak{g l}_{1} \subset L_{0} \subset W_{1}$. The general description of the Hochshild-Serre spectral sequence for relative homology (see [2]) in our case give a spectral sequence with the first page

$$
E_{p, q}^{1}=H_{q}\left(L_{0}, \mathfrak{g l}_{1}, \Lambda^{p}\left(W_{1} / L_{0}\right) \otimes S^{k} W_{1}\right)
$$

which converges to the relative homology $H_{*}\left(W_{1}, \mathfrak{g l}_{1}, S^{k} W_{1}\right)$. Let us calculate explicitly the space $E_{p, q}^{1}$. The relative homology are homology of the complex which is analogous to the Chevalley-Eilenberg complex. In our case for a fixed $p$ it is a complex

$$
\left(\Lambda^{*}\left(L_{1}\right) \otimes \Lambda^{p}\left(W_{1} / L_{0}\right) \otimes S^{k} W_{1}\right)^{g \mathfrak{g}_{1}} .
$$


It is easy to see that it can be identified with the subcomplex of all degree zero elements in the ChevalleyEilenberg complex computing the homology of $L_{1}$ with coefficients in $\Lambda^{p}\left(W_{1} / L_{0}\right) \otimes S^{k} W_{1}$. Using the results of the previous section, we obtain the complete information on the spectral sequence.

Lemma 2. The space $E_{1,1}^{1}$ is one-dimensional, and the other spaces $E_{p, q}^{1}$ are zero.

Proof. To reduce this statement to the results of the previous section, note that the $L_{1}$-modules $W_{1} / L_{0}$ and $\mathbb{C}[-1]$ (the trivial module with shifted grading) are isomorphic. Therefore $E_{1, q}^{1}=\left(E_{1, q}^{1}\right)^{(0)}=H_{q}^{(1)}\left(L_{1}, S^{k} W_{1}\right)$. Moreover, $E_{0, q}^{1}=\left(E_{0, q}^{1}\right)^{(0)}=H_{q}^{(0)}\left(L_{1}, S^{k} W_{1}\right)$. (The spaces $E_{p, q}^{1}$ for $p>1$ are obviously zero.) Now the lemma easily follows from these formulae.

Corollary 4. $E_{p, q}^{n}=E_{p, q}^{1}$.

Proof. This is true for all spectral sequences for which exactly one of the spaces $E_{p, q}^{1}$ is nonzero.

Proof of the main theorem. By Corollary 4, the space $E_{1,1}^{1}$ survives in the page $E^{\infty}$ of the spectral sequence. Since the spectral sequence converges to the desired homology, the first statement of the theorem follows.

To prove the second statement, we use the Hochshild-Serre spectral for subalgebra $\mathfrak{g l}_{1} \subset W_{1}$. The first term of this spectral sequence is

and so

$$
E_{p, q}^{1}=H_{q}\left(\mathfrak{g l}_{1}, \Lambda^{p}\left(W_{1} / \mathfrak{g l}_{1}\right) \otimes S^{k} W_{1}\right)
$$

$$
E_{p, 0}^{1}=E_{p, 1}^{1}=\left(\Lambda^{p}\left(W_{1} / \mathfrak{g I}_{1}\right) \otimes S^{k} W_{1}\right)^{\mathfrak{g}_{1}}, \quad E_{p, k}^{1}=0 \text { for } k>1 .
$$

Furthemore, the homology of $d^{1}$ for each $p=0,1$ coincide with the relative homology $H_{*}\left(W_{1}, \mathfrak{g l}_{1}, S^{k} W_{1}\right)$. It follows that the term $E^{2}$ of the spectral sequence has nonzero entries only in one column. Since the subsequent differentials $d^{k}$ map this column into other columns, they all vanish, so $E^{2}=E^{\infty}$, and the proof is complete.

\section{TENSOR POWERS}

A natural extension of this computation is the computation of the homology with coefficients in tensor powers of adjoint representation. In general, this problem seems to be rather difficult. Here we present the results of computations for the tensor powers $W_{1}^{\otimes k}$ with $1 \leq k \leq 4$, some conjectures concerning the general case, and some corollaries of our computations for homology of the current Lie algebra $W_{1} \otimes \mathbb{C}[t]$. To simplify the statements more simple, we give a more detailed discussion of the case of relative homology modulo the subalgebra $\mathfrak{g l}_{1} \subset W_{1}$ spanned by $e_{0}$.

Consider the mapping

$$
H_{*}\left(\mathfrak{s l}_{2}, \mathfrak{g l}_{1}, \mathbb{C}\right) \otimes\left(\mathfrak{s l}_{2}^{\otimes n}\right)^{\mathfrak{s} \mathfrak{l}_{2}}=H_{*}\left(\mathfrak{s l}_{2}, \mathfrak{g l}_{1}, \mathfrak{s l}_{2}^{\otimes n}\right) \rightarrow H_{*}\left(W_{1}, \mathfrak{g l}_{1}, W_{1}^{\otimes n}\right),
$$

induced by the embedding $\mathfrak{s l}_{2} \hookrightarrow W_{1}$. The homology of $\mathfrak{s l}_{2}$ modulo $\mathfrak{g l}_{1}$ is zero, except for $H_{0}$ and $H_{2}$. Thus to describe this mapping it suffices to consider only the zero and second homology.

Proposition 4. The mapping (2) is zero on the space $\mathrm{H}_{0}$ and is an embedding on the space $\mathrm{H}_{2}$.

Proof. The first assertion is obvious, since $H_{0}\left(W_{1}, \mathfrak{g l}_{1}, W_{1}^{\otimes n}\right)=0$; in fact, already $W_{1}^{\otimes n} / e_{-1}\left(W_{1}^{\otimes n}\right)=0$. Consider the second homology. Let

$$
e_{-1} \wedge e_{1} \otimes \psi=d\left(\sum_{0<\alpha<\beta} e_{-1} \wedge e_{\alpha} \wedge e_{\beta} \otimes \psi_{\alpha \beta}+\sum_{0<\alpha<\beta<\gamma} e_{\alpha} \wedge e_{\beta} \wedge e_{\gamma} \otimes \psi_{\alpha \beta \gamma}\right) .
$$

Then $\psi=\sum_{1<\beta} e_{\beta}\left(\psi_{1 \beta}\right)$, which results in a contradiction, since $\psi$ is an $\mathfrak{s l}_{2}$-invariant element in $\mathfrak{s l}_{2}^{\otimes n}$, and $e_{\beta}\left(W_{1}^{\otimes n}\right) \cap\left(\mathfrak{s l}_{2}^{\otimes n}\right)^{\mathfrak{s l}}=0$ : for $\beta>2$ or $\beta=2, n>2$ the elements from $e_{\beta}\left(W_{1}^{\otimes n}\right)$ do not even belong to $\mathfrak{s l}_{2}^{\otimes n}$, and for $\beta=n=2$ the intersection with the tensor power of $\mathfrak{s I}_{2}$ is one-dimensional, and the corresponding element $e_{2}\left(e_{-1}^{2}\right)$ is not invariant. 
Remark 1. We believe that the thus obtained lower bound for the second homology is almost sharp. Of course, for $k=2 m+1$ the main theorem ensures that $\operatorname{dim} H_{2}\left(W_{1}, \mathfrak{g l}_{1}, S^{k} W_{1}\right)=1$, while $\operatorname{dim} H_{2}\left(\mathfrak{s l}_{2}, \mathfrak{g l}_{1}, S^{k} \mathfrak{s l}_{2}\right)=0$. However, all cycles known so far to lie outside the image of the embedding constructed here are the cycles $\psi_{2 k-1}, k=1,2, \ldots$ for odd symmetric powers.

For $1 \leq k \leq 4$, an explicit calculation shows that the relative homology with coefficients in $W_{1}^{\otimes n}$ is exhausted by the image of embedding from Proposition 4 and the homology with coefficients in symmetric powers. The results of this computation are gathered in the following proposition. The proof is technical and therefore omitted.

Proposition 5. $H_{i}\left(W_{1}, \mathfrak{g l}_{1}, W_{1}^{\otimes k}\right)=0$ for $i \neq 2,1 \leq k \leq 4$. Furthermore,

$$
\begin{gathered}
H_{2}\left(W_{1}, \mathfrak{g l}_{1}, W_{1}\right)=H_{2}\left(\mathfrak{s l}_{2}, \mathfrak{g l}_{1}, \mathfrak{s l}_{2}\right) \oplus H_{2}\left(W_{1}, \mathfrak{g l}_{1}, S^{1} W_{1}\right)=\mathbb{C}^{0} \oplus \mathbb{C}^{1}=\mathbb{C}, \\
H_{2}\left(W_{1}, \mathfrak{g l}_{1}, W_{1}^{\otimes 2}\right)=H_{2}\left(\mathfrak{s l}_{2}, \mathfrak{g l}_{1}, \mathfrak{s l}_{2}^{\otimes 2}\right)=\mathbb{C}, \\
H_{2}\left(W_{1}, \mathfrak{g l}_{1}, W_{1}^{\otimes 3}\right)=H_{2}\left(\mathfrak{s l}_{2}, \mathfrak{g l}_{1}, \mathfrak{s l}_{2}^{\otimes 3}\right) \oplus H_{2}\left(W_{1}, \mathfrak{g l}_{1}, S^{3} W_{1}\right)=\mathbb{C}^{1} \oplus \mathbb{C}^{1}=\mathbb{C}^{2}, \\
H_{2}\left(W_{1}, \mathfrak{g l}_{1}, W_{1}^{\otimes 4}\right)=H_{2}\left(\mathfrak{s l}_{2}, \mathfrak{g l}_{1}, \mathfrak{s l}_{2}^{\otimes 4}\right)=\mathbb{C}^{3} .
\end{gathered}
$$

Corollary 5. $H_{i}\left(W_{1}, W_{1}^{\otimes k}\right)=0$ for $i \neq 2,3,1 \leq k \leq 4$. Furthermore,

$$
\begin{gathered}
H_{2}\left(W_{1}, W_{1}\right)=H_{3}\left(W_{1}, W_{1}\right)=\mathbb{C}, \\
H_{2}\left(W_{1}, W_{1}^{\otimes 2}\right)=H_{3}\left(W_{1}, W_{1}^{\otimes 2}\right)=\mathbb{C}, \\
H_{2}\left(W_{1}, W_{1}^{\otimes 3}\right)=H_{3}\left(W_{1}, W_{1}^{\otimes 3}\right)=\mathbb{C}^{2}, \\
H_{2}\left(W_{1}, W_{1}^{\otimes 4}\right)=H_{3}\left(W_{1}, W_{1}^{\otimes 4}\right)=\mathbb{C}^{3} .
\end{gathered}
$$

Conjecture. For $i>2$ and all $k$, we have $H_{i}\left(W_{1}, \mathfrak{g l} l_{1}, W_{1}^{\otimes k}\right)=0$; for $i>3$ and all $k$, we have $H_{i}\left(W_{1}, W_{1}^{\otimes k}\right)=0$.

Proposition 5 and Corollary 5 suggest that even the stronger conjecture replacing the condition $i>2$ (respectively, $i>3$ ) by $i \neq 2$ (respectively, $i \neq 2,3$ ) might be true. It turns out that this is not the case: for example, the first homology is not zero for $W_{1}^{\otimes 5}$. Indeed, let us consider the action of the symmetric group $S_{5}$ on $W_{1}^{\otimes 5}$ by permutations of the factors. The decomposition of the tensor power into the direct sum of isotypic components is known: this representation is obviously isomorphic to the representation in the space of all polynomials in five variables (the gradings of these vector spaces differ only by a shift), and the results on the decomposition of latter representation are standard. For the representation of $S_{5}$ corresponding to the partition $5=2+1+1+1$, the minimal degree of homogeneous polynomials in five variables where that representation occurs is equal to 6; moreover, this representation occurs in the subspace of polynomials of degree 6 with multiplicity 1 . Shifting the grading, we see that for $W_{1}^{\otimes 5}$ this representation occurs for the first time in degree 1 with multiplicity 1 . Denote by $V$ the corresponding subspace. Then it is easy to see that any element of the space $e_{-1} \otimes V$ is a cycle in the Chevalley-Eilenberg complex (both the relative one and the ordinary one) which is nontrivial, since this representation does not occur in lower degrees.

Information on the homology with coefficients in tensor powers is useful in that it can be used for homology computations for the Lie algebra of (polynomial) currents on a line with values in $W_{1}$. Recall that the algebra of polynomial currents on a line with values in a Lie algebra $\mathfrak{A}$ is the vector space $\mathfrak{A} \otimes \mathbb{C}[t]$ equipped with a bracket $[a \otimes f, b \otimes g]=[a, b] \otimes(f g)$. The computation uses the Hochshild-Serre spectral sequence modulo the subalgebra $W_{1} \otimes 1$. We list here without a proof the results on the relative homology modulo the subalgebra $\mathfrak{g l}_{1}:=\mathbb{C} e_{0} \otimes 1 \subset W_{1} \otimes 1 \subset W_{1} \otimes \mathbb{C}[t]$.

It is convenient to state the results as follows. The Lie algebra of vector fields on the $t$-line acts on the current algebra by an obvious rule $\xi(a \otimes f)=a \otimes \xi(f)$. This action can be extended in a standard way to all tensor constructions, for example, to exterior powers and to the entire Chevalley-Eilenberg. Obviously, this action commutes with the boundary maps, and so the Lie algebra of vector fields on the line acts on the homology. 


\section{Proposition 6.}

$$
\begin{aligned}
& H_{0}\left(W_{1} \otimes \mathbb{C}[t], \mathfrak{g l}_{1}, \mathbb{C}\right) \cong \mathbb{C} \\
& H_{1}\left(W_{1} \otimes \mathbb{C}[t], \mathfrak{g l}_{1}, \mathbb{C}\right) \cong 0, \\
& H_{2}\left(W_{1} \otimes \mathbb{C}[t], \mathfrak{g l}_{1}, \mathbb{C}\right) \cong \mathbb{C} \\
& H_{3}\left(W_{1} \otimes \mathbb{C}[t], \mathfrak{g l}_{1}, \mathbb{C}\right) \cong \mathbb{C}[t] d t, \\
& H_{4}\left(W_{1} \otimes \mathbb{C}[t], \mathfrak{g l}_{1}, \mathbb{C}\right) \cong 0, \\
& H_{5}\left(W_{1} \otimes \mathbb{C}[t], \mathfrak{g l}_{1}, \mathbb{C}\right) \cong S^{3}(\mathbb{C}[t] d t)
\end{aligned}
$$

These formulas should be interpreted as isomorphisms of modules over the Lie algebra of vector fields on the line.

\section{REFERENCES}

[1] B. L. Feigin and B. L. Tsygan, "Riemann-Roch theorem and Lie algebra cohomology," In: Proc. of the Winter School on Geometry and Physics (Srni, 9-16 January 1988), Rend. Circ. Mat. Palermo (2) Suppl., No. 21, 15-52 (1989).

[2] D. B. Fuks, Cohomology of Infinite-Dimensional Lie Algebras, Consultants Bureau, New York, 1986

[3] H. Cartan and S. Eilenberg, Homological algebra, Princeton University Press, Princeton, 1956.

INDEPENDENT UNIVERSITY OF Moscow

E-mail address: dotsenko@mccme.ru 